\title{
ITPP Treatment of RG2 Glioblastoma in a Rat Model
}

\author{
KAROLINA FÖRNVIK, SHAIAN ZOLFAGHARI, LEIF G. SALFORD and HENRIETTA NITTBY REDEBRANDT \\ The Rausing Laboratory, Division of Neurosurgery, \\ Department of Clinical Sciences Lund, Lund University, Lund, Sweden
}

\begin{abstract}
Background: Inositol trispyrophosphate (ITPP) has been shown to reduce tumour growth in different animal cancer models, as well as of human U87 glioma cells grafted onto chick chorioallantoic membrane (CAM). The aim of this study was to establish whether ITPP crosses the blood-brain barrier and whether it halts the growth of RG2 glioblastoma tumour. Materials and Methods: A model comprising of Fischer 344 rats was chosen and RG2 cells were implanted either intracranially, or subcutaneously on the left hind leg, and the animals were treated with ITPP either intraperitoneally, intravenously or both routes combined. Overall survival was then calculated. Results: No prolonged survival was seen in animals treated with ITPP. The route of ITPP administration did not affect outcome. Conclusion: ITPP had no favourable effect upon survival in our animal model with RG2 glioblastoma tumours in Fischer 344 rats.
\end{abstract}

Glioblastoma multiforme (GBM) is the most common and aggressive type of primary brain tumour in adults. There are currently no curative ways to treat these malignant tumours, even though patients undergo multimodality treatment with surgery, chemotherapy and radiotherapy. One of the key problems when treating patients with malignant gliomas is that despite being able to remove the major bulk of the tumour through neurosurgery, it is known that malignant tumour cells have already spread throughout the brain and even extensive resections will not cure the patient (1).

One way of limiting tumour growth could theoretically be by controlling angiogenesis, since a major hallmark of malignant growth is the rapid formation of new blood vessels

This article is freely accessible online.

Correspondence to: Karolina Förnvik, The Rausing Laboratory, Division of Neurosurgery, Department of Clinical Sciences Lund, Lund University, SE-221 85 Lund, Sweden. Tel.: +46 704437847, e-mail: karolina.fornvik@med.lu.se

Key Words: Glioblastoma multiforme, RG2, hypoxia, inositol trispyrophosphate, angiogenesis. in an unstructured manner. These vessels are permeable and allow tumour spreading. The driving force is thought to be the hypoxic state created by the tumour itself (2). It has been shown that inositol trispyrophosphate (ITPP) treatment increases oxygen delivery to the tumour and reduces tumour growth, thus resulting in a more favourable outcome in animal models with pancreatic cancer (3), liver cancer (4) and glioblastoma cells implanted on membranes (5). These effects may result from the increased oxygen delivery caused by ITPP shifting the oxygen binding affinity of haemoglobin.

2,3-Bisphosphoglyceric acid (BPG) is found in red blood cells (RBCs) and is the natural allosteric effector that reduces the oxygen-binding affinity of human haemoglobin and thus facilitates the release of oxygen by RBCs in tissue that needs it the most (6). 2,3-BPG can help prevent tissue hypoxia in conditions where it is likely to occur, such as at high altitude, and it has been shown that people acclimated to high altitudes have higher levels of 2,3-BPG (6). The same is true in pregnant women, in whom there is an increase in 2,3-BPG (7). This leads to a decrease in the haemoglobin affinity for oxygen in the mother, and thus allows oxygen to be delivered to the growing foetus through the maternal uterine arteries where the oxygen pressure is low (7).

Inositol hexakisphosphate (IP6), also called phytic acid, can be found in many plants, where it acts as the principal storage form of phosphorus (8). It is also produced in mammalian cells and has antioxidative properties. IP6 has been shown to be a powerful allosteric effector of haemoglobin and increases the regulated oxygen-releasing capacity of RBCs. IP6 would therefore be a good candidate for a drug to increase oxygen levels in hypoxic tissue, but unfortunately IP6 does not cross the RBC membrane (9). Fylaktakidou et al. synthesised a molecule based on IP6 that does in fact interact with and possibly cross the RBC membrane. It is called myo-inositol trispyrophosphate (ITPP) (9). ITPP increases the oxygen level and blood flow in experimental settings of melanoma and breast cancer models (2). ITPP also inhibited angiogenesis in chick chorioallantoic membrane (CAM) (5). Furthermore, it was reported that after 2 days of ITPP treatment of human U87 glioma cell nodules grafted onto CAM, there was a reduction of the number of 
neovessels. ITPP had no effect on U87 cell proliferation in vitro. In another study by Kieda et al., it was described that ITPP treatment reduced the size of malignant melanomas in mice and reduced the metastatic invasion, leading to increased survival compared to control animals (2).

The mechanism of action of ITPP is suggested to be through regulation of hypoxia-inducible factors (HIFs). ITPP reduces HIF $1 \alpha$ mRNA expression (2). HIF1 $\alpha$ is associated with a higher number of vessels, tumour grade severity and treatment failure (2). It has been suggested that hypoxic tumour cells are resistant to radiotherapy and chemotherapy through HIFs and it is thought that counteracting hypoxia in the tumour by normalizing tumour vessels could improve the results of the radiotherapy (2).

Murine lung endothelial cells showed a reorganisation of phosphatase and tensin homolog (PTEN) in the presence of ITPP (2). PTEN is one of the most commonly lost tumour suppressors in human cancer and inactivation of PTEN occurs in glioblastoma, endometrial cancer, and prostate cancer; and reduced expression is found in many other tumour types such as lung and breast cancer (10). It has also been shown that patients with defective PTEN can develop cerebellar mass lesions called dysplastic gangliocytomas or Lhermitte-Duclos disease (11).

The aim of the current investigation was to establish whether ITPP halts the growth of RG2 glioblastoma tumours in a similar fashion to U87 glioma cells on CAMs and subcutaneous malignant melanomas in Balb mice. Since it is well known that glioblastomas have both angiogenesis and pathological vessels, as well as a hypoxic environment, we hypothesized that ITPP treatment could result in a more favourable outcome. A model comprising female Fischer 344 rats was chosen and RG2 cells were implanted either intracranially in the right caudate nucleus or subcutaneously on the left hind leg and the animals were treated with ITPP. The RG2 model was chosen since this is a well-established glioblastoma model used in many previous animal studies (12). ITPP was administered by different routes (intraperitoneal, intravenous and both routes combined). Subcutaneous tumour implantation was used in order to evaluate whether the bloodbrain barrier (BBB) affected the response to ITPP or not, since it is well known that the $\mathrm{BBB}$ restricts the passage of molecules from the blood circulation into the brain parenchyma, although the BBB is partially disturbed around malignant tumours in the brain.

\section{Materials and Methods}

Animals. A total of 37 female Fischer 344 rat, were included in this study. They were housed two per cage with tap water and rodent chow ad libitum. The procedures performed on the animals were approved by the Animal Ethics Committee in Lund (permit number M50-10). Rats were obtained from Charles River Laboratories, Germany.
Cell line. The rat glioma cell line RG2 was originally derived from ethylnitrosurea-induced glioma in Fischer 344 rats. The RG2 cells in this study were cultured in RPMI-1640 medium (Sigma Aldrich) supplemented with $5 \mathrm{ml} \mathrm{Na-pyruvate} \mathrm{(100} \mathrm{mM} \mathrm{stock} \mathrm{solution)} \mathrm{and}$ $5 \mathrm{ml}$ Hepes (1 M stock solution). Gentamycin $(0.5 \mathrm{ml}$ of $50 \mathrm{mg} / \mathrm{ml})$ was added to the nutrient medium to avoid infection.

Inoculation. The cell lines were inoculated in such a manner as to investigate whether or not the BBB had an effect on the efficacy of ITPP. One group were inoculated subcutaneously on the right hind leg $(n=10)$, while the other was inoculated intracranially $(n=27)$. The rats were sedated with Isoflurane during the procedure.

The intracranial procedure. The rats were sedated and fixated with a stereotactic frame and a hole was drilled 1-2 mm laterally to the sagittal suture, at the level of the coronal suture on the right side. A total of $5000 \mathrm{RG} 2$ cells were inoculated at a depth of $5 \mathrm{~mm}$ from the skull corresponding to the location of the caudate nucleus.

The subcutaneous procedure. The rats were injected with $10^{5} \mathrm{RG} 2$ cells subcutaneously in the right hind leg.

Treatment. The rats were administered ITPP (kindly provided by Professor Jean-Marie Lehn) prior to symptoms. ITPP was administered either through an intraperitoneal route (i.p.) $(1.5 \mathrm{~g} / \mathrm{kg}$ in saline) or an intravenous (i.v.) route. The drug was administered on days $7,8,15,16,21,22,28$ and 29 after tumour cell inoculation. The dose of $1.5 \mathrm{~g} / \mathrm{kg}$ was chosen since this dose had been used in models of pancreatic, melanoma and prostate cancer (2). Treatment was to be cancelled if any of the following occurred: neurological deficits such as paresis and circling, impaired general condition (weight loss, shaggy fur, red eyes), a tumour diameter of $3 \mathrm{~cm}$, or a volume of $9 \mathrm{~cm}^{3}$ regarding the subcutaneous tumours. If none of these criteria were met, the rats would be observed for a total of 100 days. After 100 days, the rats would be euthanized.

The 37 rats were divided into six different groups: six rats with intracranial tumours received i.v. ITPP; six rats with intracranial tumours received i.v. and i.p. ITPP; six rats with intracranial tumours received i.p. ITPP; six rats with intracranial tumours were untreated controls; five rats with subcutaneous tumours received i.p. ITPP; and five animals with subcutaneous tumours were untreated controls. Three additional animals with intracranial tumours were treated with ITPP the first day after tumour inoculation, with the same dose of $1.5 \mathrm{~g} / \mathrm{kg}$ in saline.

Statistics. Overall survival was demonstrated with Kaplan-Meier curves. Differences in survival were analysed with Mann-Whitney $U$-test for the groups with subcutaneous tumours (two groups) and the Kruskal-Wallis test for those with intracranial tumours (four groups), using ExcelStat (Addisonsoft, Microsoft Excel).

\section{Results}

The rats with subcutaneous tumours each received eight treatments with ITPP. This was more compared to the rats with intracranial tumours, which received 3-5 treatments, because the rats with the intracranial tumours died earlier and therefore could not receive the same number of treatments. 


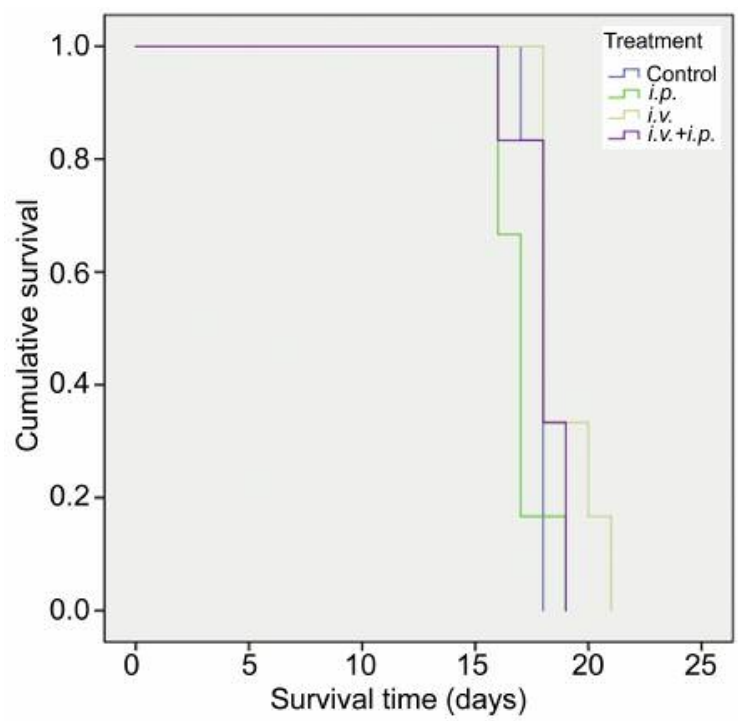

Figure 1. Kaplan-Meier analysis of rat survival after intracranial inoculation of $R G 2$ cells and treatment with intraperitoneal (i.p.) inositol trispyrophosphate (ITPP), intravenous (i.v.) ITPP; or combined i.v. and i.p. ITPP.

No effect on survival of animals with intracranial tumours. All rats with intracranial tumours, both the ITPP-treated and the controls, died or had to be euthanized on day 17-21 (Figure 1). This survival time is in line with historical data for RG2 tumour development in untreated rats (12).

When comparing all five groups with intracranial tumours, a negative effect was seen on the animals which received ITPP treatment on the first day after tumour inoculation (Kruskal-Wallis, $p=0.009$ for all groups, and MannWhitney, $p=0.001$ comparing this group to the control animals). The rats which received ITPP on the first day post surgery died on days 15,15 and 16, respectively.

There was no difference in survival of ITPP-treated rats compared to the control rats for the animals which received their first treatment one week after tumour inoculation, and the route of ITPP administration (i.v. versus i.p. versus i.v. plus i.p.) did not affect outcome (Mann-Whitney, $p>0.05$ ).

No effect on survival of animals with subcutaneous tumours. Three control animals with subcutaneous tumours were euthanised on day 100 , since the ethical permission did not allow for longer observation. These three rats did not have a tumour size exceeding the limit of $3 \mathrm{~cm}$ in diameter. In the rats with subcutaneous tumours, it seems the ITPP negatively affected tumour control, and the survival was greater for the control group as compared to the ITPP-treated group, however, this difference was not significant (Mann-Whitney $p=0.09$ ) (Figure 2).

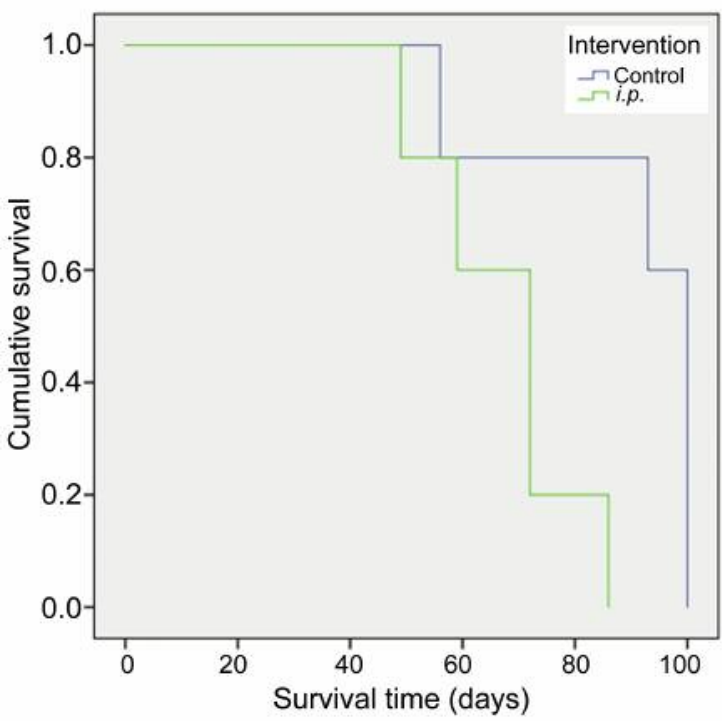

Figure 2. Kaplan-Meier analysis of rat survival after subcutaneous inoculation of RG2 cells and treatment with intraperitoneal (i.p.) inositol trispyrophosphate (ITPP).

\section{Discussion}

ITPP has been shown to reduce tumour growth in various types of cancer models in rats, such as hepatocellular carcinoma (4) and pancreatic cancer (3). It was also effective against growth of U87 glioma cells in a CAM model (5). Preliminary data from Coquery et al. at the Grenoble Institute of Neurosciences using ITPP to treat F98 glioma in rats point towards reduced tumour growth after one injection and they also saw a tendency for increased tissue oxygen saturation on T1-weighted magnetic resonance images, increased cerebral blood volume and increased apparent diffusion constant (personal communication, 2013).

To our surprise, ITPP had no effect against RG2 glioma cells as our results show, with no effect on survival. Previously other groups have had negative results concerning the influence of ITPP on growth of C6 glioma tumour in rats (Nicolau et al. personal communication). Since the results show no significant difference between ITPP treatment of intracranial tumours and subcutaneous tumours, we are unable to draw any conclusions about ITPP overcoming the restrictions of the BBB. The only effect that was seen was that very early ITPP treatment starting as early as the day after tumour inoculation affected survival negatively.

Myo-inositol on the other hand is well known to cross the $\mathrm{BBB}$ and is already in use for the treatment of several psychiatric conditions. There are studies reporting polymer nanoparticles penetrating the $\mathrm{BBB}$ in healthy brain when 
delivered by convection-enhanced delivery, and nanoparticle size has been shown to be a critical factor for penetration (13).

There are some significant differences between the RG2 and U87 glioma cell lines. RG2 is a particularly aggressive model with very quick growth leading to a very short survival time (19.4 \pm 3.8 days) compared to other rodent glioma models such as $\mathrm{C6}$ or 9L and also compared to human-derived U87 $(12,14)$. However, one advantage of the RG2 model is the stable development of tumours, as described by Aas et al. (12), and that it can be used in fully immunocompetent animals.

Of the commonly used rat glioma models (C6, 9L, T9, RG2, F98, BT4C, RT-2 and CNS-1) the 9L and T9 gliomas are induced by repeated injections of methylnitrosourea to adult Fischer rats, whereas RG2 and F98 are induced by administering ethylnitrosourea to pregnant Fischer rats, the progeny of which developed brain tumours $(12,15)$. T9 is essentially the same as $9 \mathrm{~L}$ and can be immunogenic in syngeneic hosts. RG2 and F98 on the other hand are either weakly or non-immunogenic and have a very invasive pattern of growth and uniform lethality, making them the model of choice when it comes to testing new therapeutic modalities. RG2 has increased expression of platelet-derived growth factor subunit B, insulin-like growth factor-1, receptor tyrosine-protein kinase ERBB-3, precursor mRNA and cyclin D2 (15).

ITPP is purported to exert its effect by restoring normoxia in the tumour and reducing HIF1 $\alpha$ levels, with subsequent down-regulation of vascular endothelial growth factor and lysyl oxydase, which results in improved vessel structure (2). Ineffective vasculature can block the access of immune cells to the tumour and ITPP has also been shown to increase the percentage of macrophages $\left(\mathrm{F} 4 / 80^{+}\right)$and $\mathrm{CD}^{+} \mathrm{T}$ lymphocyte subpopulations in the tumours as compared to controls.

Glioblastoma multiforme is also very hard to treat, when trying to target the abnormal vasculature. In recent years, much effort has been put into patient studies trying to find inhibitors of VEGF receptors, but no effect upon survival was demonstrated despite well-conducted studies with large patient cohorts (1).

McCord et al. grew tumour stem-like cells isolated from human glioblastoma at $7 \%$ and $20 \%$ oxygen concentration. They found that growing the cells at the more physiologically relevant level of $7 \%$ oxygen reduced their doubling time and increased the self-renewal potential as reflected by clonogenicity. The cultures also expressed higher levels of HIF $2 \alpha$ and exhibited an enhanced capacity to differentiate along both the glial and neuronal pathways (16). One possible explanation for ITPP having a negative effect in our model could therefore be that the already aggressive RG2 cells start dividing even more rapidly when normoxia is restored by ITPP.
It is not clear, however, that hypoxia actually favours tumour growth. Steinbach et al. describe that in human malignant glioma cells, hypoxia leads to de-coupling of mitochondrial cytochrome $c$ release from caspase and necrotic cell death. However, if normoxia is restored within $8 \mathrm{~h}$, ATP levels that had declined could return to normal values. Furthermore, they described how tumour hypoxia during tumour development leads to the selection of hypoxia-resistant clones (17).

Another aspect is that glioblastoma cells seem to adapt to hypoxia. Kucharzewska et al. showed that secreted vesicles with exosome characteristics mediate hypoxia-dependent intercellular signalling of glioblastoma multiforme. Endothelial cells were programmed by glioblastoma multiforme cell-derived hypoxic exosomes to secrete several potent growth factors and cytokines and to stimulate pericyte hosphatidylinositol-4,5-bisphosphate 3-kinase/protein kinase $\mathrm{B}$ signalling activation and migration. The findings in the hypoxic glioblastoma cells were correlated to enhanced induction by hypoxic compared to normoxic exosomes of tumour vascularization, pericyte vessel coverage, glioblastoma multiforme cell proliferation, as well as decreased tumour hypoxia in a mouse xenograft model (18).

\section{Conclusion}

From this study, we conclude that ITPP had no favourable effect upon survival in our animal model with RG2 glioblastoma tumours in Fischer 344 rats. This was quite surprising, since ITPP had positive effects in other models with hypoxic tumours. One theory could be that the glioblastoma cells, which are very adaptive, benefited from the extra oxygen supply, which our subcutaneous tumour study also indicates by the more rapid death of animals which received ITPP on the first day after tumour inoculation. This could also be in line with extensive studies on VEGF receptor inhibition in patients with glioblastoma, where no effect upon survival was found. With this study we would also like to point out the importance of performing studies in fully immunocompetent animals, since it is well know that the immune response is a key in the glioblastoma setting.

\section{Acknowledgements}

The Authors would like to thank Professor Jean-Marie Lehn for initiating this study and for supplying the ITPP and Professor Claude Nicolau for constructive discussions. We would also like to thank MD Jonatan Ahlstedt for technical assistance during the subcutaneous experiments.

\section{References}

1 Weathers S-P and de Groot J: VEGF Manipulation in glioblastoma. Oncology 29: 720-727, 2015. 
2 Kieda C, El Hafny-Rahbi B, Collet G, Lamerant-Fayel N, Grillon C, Guichard A, Dulak J, Jozkowicz A, Kotlinowski J, Fylaktakidou KC, Vidal A, Auzeloux P, Miot-Noirault E, Beloeil J-C, Lehn J-M and Nicolau C: Stable tumor vessel normalization with $\mathrm{pO}_{2}$ increase and endothelial PTEN activation by inositol trispyrophosphate brings novel tumor treatment. J Mol Med 91: 883-899, 2013.

3 Raykov Z, Grekova SP, Bour G, Lehn JM, Giese NA, Nicolau $\mathrm{C}$ and Aprahamian M: Myo-inositol trispyrophosphate-mediated hypoxia reversion controls pancreatic cancer in rodents and enhances gemcitabine efficacy. Int J Cancer 134: 2572-2582, 2014.

4 Aprahamian M, Bour G, Akladios CY, Fylaktakidou K, Greferath R, Soler L, Marescaux J, Egly J-M, Lehn J-M and Nicolau C: Myo-inositoltrispyrophosphate treatment leads to HIF-1 $\alpha$ suppression and eradication of early hepatoma tumors in rats. Chembiochem 12: 777-783, 2011.

5 Sihn G, Walter T, Klein J-C, Queguiner I, Iwao H, Nicolau C, Lehn J-M, Corvol P and Gasc J-M: Anti-angiogenic properties of myo-inositol trispyrophosphate in ovo and growth reduction of implanted glioma. FEBS Lett 581: 962-966, 2007.

6 Biolo A, Greferath R, Siwik DA, Qin F, Valsky E, Fylaktakidou KC, Pothukanuri S, Duarte CD, Schwarz RP, Lehn J-M, Nicolau $\mathrm{C}$ and Colucci WS: Enhanced exercise capacity in mice with severe heart failure treated with an allosteric effector of hemoglobin, myo-inositol trispyrophosphate. Proc Natl Acad Sci USA 106: 1926-1929, 2009.

7 Pritlove DC, Gu M, Boyd C a. R, Randeva HS and Vatish M: Novel placental expression of 2,3-bisphosphoglycerate mutase. Placenta 27: 924-927, 2006.

8 Irvine RF and Schell MJ: Back in the water: the return of the inositol phosphates. Nat Rev Mol Cell Biol 2: 327-338, 2001.

9 Fylaktakidou KC, Lehn J-M, Greferath R and Nicolau C: Inositol tripyrophosphate: a new membrane permeant allosteric effector of haemoglobin. Bioorg Med Chem Lett 15: 1605-1608, 2005.

10 Yin Y and Shen WH: PTEN: a new guardian of the genome. Oncogene 27: 5443-5453, 2008.
11 Pilarski R and Eng C: Will the real Cowden syndrome please stand up (again)? Expanding mutational and clinical spectra of the PTEN hamartoma tumour syndrome. J Med Genet 41: 323326, 2004.

12 Aas AT, Brun A, Blennow C, Strömblad S and Salford LG: The RG2 rat glioma model. J Neurooncol 23: 175-183, 1995.

13 Saucier-Sawyer JK, Seo Y-E, Gaudin A, Quijano E, Song E, Sawyer AJ, Deng Y, Huttner A and Saltzman WM: Distribution of polymer nanoparticles by convection-enhanced delivery to brain tumors. J Control Release 232: 103-112, 2016.

14 Doblas S, He T, Saunders D, Hoyle J, Smith N, Pye Q, Lerner $\mathrm{M}$, Jensen RL and Towner RA: In vivo characterization of several rodent glioma models by $1 \mathrm{H}$ MRS. NMR Biomed 25: 685-694, 2012.

15 Barth RF and Kaur B: Rat brain tumor models in experimental neuro-oncology: the C6, 9L, T9, RG2, F98, BT4C, RT-2 and CNS-1 gliomas. J Neurooncol 94: 299-312, 2009.

16 McCord AM, Jamal M, Shankavaram UT, Shankavarum UT, Lang FF, Camphausen K and Tofilon PJ: Physiologic oxygen concentration enhances the stem-like properties of CD133+ human glioblastoma cells in vitro. Mol Cancer Res 7: 489-497, 2009.

17 Steinbach JP, Wolburg H, Klumpp A, Probst H and Weller M: Hypoxia-induced cell death in human malignant glioma cells: energy deprivation promotes decoupling of mitochondrial cytochrome c release from caspase processing and necrotic cell death. Cell Death Differ 10: 823-832, 2003.

18 Kucharzewska P, Christianson HC, Welch JE, Svensson KJ, Fredlund E, Ringnér M, Mörgelin M, Bourseau-Guilmain E, Bengzon $\mathrm{J}$ and Belting M: Exosomes reflect the hypoxic status of glioma cells and mediate hypoxia-dependent activation of vascular cells during tumor development. Proc Natl Acad Sci USA 110: 7312-7317, 2013.
Received July 3, 2016

Revised July 18, 2016

Accepted July 21, 2016 